\title{
A morphometric study of the thoracolumbar spine spinous process and lamina space in the Chinese
}

\author{
L.N. Leng ${ }^{1,2}$, H.J. $\mathrm{Ma}^{3,4 *}$, D.W. Si ${ }^{5 *}$ \\ 'Graduate School, HeBei North University, Zhangjiakou, Hebei Province, China \\ ${ }^{2}$ Hebei General Hospital, Shijiazhuang, Hebei, China \\ ${ }^{3} \mathrm{HeBei}$ North University, Zhangjiakou, Hebei Province, China \\ ${ }^{4}$ Hebei Key Laboratory of Metabolic Diseases, Hebei General Hospital, Shijiazhuang, Hebei, China \\ ${ }^{5}$ Department of Anatomy of College of Basic Medical Sciences, North China University of Science and Technology, \\ Tangshan, Hebei Province, China
}

[Received: 27 April 2020; Accepted: 22 July 2020; Early publication date: 24 August 2020]

Background: The aim of the study was to analyse the anatomical parameters of the thoracolumbar spine spinous process and lamina space for Chinese anatomic study, and provide an anatomical reference for its clinical operation.

Materials and methods: Samples from 24 adult autopsy subjects were obtained from the thoracolumbar spine spinous process and lamina space at levels $T 1$ through $L 5$. Direct measurements were made for the spinous process spacing distance, spinous process length, width, thickness and inclination angle, and the lamina space width and height.

Results: 1. Distance of the spine spinous process spacing: Thoracic part: The maximum tip distance was observed at T4 T5 level, and the minimum tip distance was observed at T9 T10 level. The maximum centre distance and root distance were observed at T11 T12 level, and the minimum were observed at T5 T6 level separately. Lumbar part: distance of spinous process spacing in lumbar part showed a decreasing pattern from $L 1 \sim L 2$ to $L 5 \sim S 1$. 2. Length, width, thickness of the spine spinous process: 1) The length of the spinous process: The upper border gradually increased from T1 to T6 and then decreased till T12 region. The centre region is T8 maximum, T11 minimum. The lower border length showed a decreasing trend from $T 1$ to T12. Lumbar part: The length increased from $L 1$ and reached maximum value at $L 3$. Then, the length decreased gradually to reach minimum value at L5. 2) The width of the spinous process: The width showed an increasing trend from $T 1$ to T12. Lumbar part: Maximum width was seen at $L 3$ and a minimum L5. 3) The thickness of the spinous process: Tip thickness $>$ Centre thickness $>$ Root thickness in each thoracic and lumbar vertebra. Thoracic part: the maximum tip thickness is $T 1, T 7$ minimum, The maximum centre thickness is T12, $T 7$ minimum. The maximum root height is T6, T9 minimum. Lumbar part: Maximum tip thickness was seen at $L 1$, and a minimum $L 3$. Maximum centre thickness was seen at L5, and a minimum L2. Maximum root thickness was seen at $L 2$, and a minimum $L 1$. 3. Inclination angle of the spine spinous process: The

Address for correspondence: Dr. H.J. Ma, HeBei North University, Zhangjiakou 075000, Hebei Province, China; Hebei Key Laboratory of Metabolic Diseases, Hebei General Hospital, 348 Heping West Road, Xinhua District, Shijiazhuang 050051, Hebei, China, e-mail: huijuanma76@163.com 
inclination angle gradually decreased from $T 1$ to $T 7$ to minimum value at $T 7$ and then increased till $T 12$ region. 4. Width and height of lamina space: 1) The width of lamina space: For thoracic part, the data became shorter gradually from T1 T2 to T5 T6, and then increased till to T11 T12. For lumbar part, the width of lamina space increased from $T 12 \sim L 1$ to $L 5 \sim S 1$. 2) The height of lamina space: In the thoracic vertebrae, the maximum height of centre region was observed at T11 T12 and the minimum mean value was observed at T3 T4. In the lumbar vertebrae, the height of the lamina space was gradually increased from $T 12 \sim L 1$ to $L 5 \sim S 1$.

Conclusions: This study reports morphometric data of the thoracolumbar spine spinous process and lamina space in the Chinese population, which provides an anatomic basis for thoracolumbar spine design of internal fixation, posterior surgery, puncture and epidural anaesthesia. (Folia Morphol 2021; 80, 3: 665-674)

Key words: thoracolumbar spinous process, lamina space, anatomy, morphology

\section{INTRODUCTION}

Lumbar spinous processes (SPs) are an appealing target for applications in spine surgery, which are relatively superficial and easy to access anatomically. Currently, a number of devices and techniques use lumbar SPs or the lamina space for instrumentation $[2,11,16-18,30,39]$. Thoracic part of vertebral column is even more complex $[8,9,29,33]$. Knowledge of morphology of the thoracic spine is essential for the anaesthetic and surgical procedures carried out in this part of the vertebral column, to achieve desired results and to avoid complications. Thoracic epidural anaesthesia and pedicle screw fixation of thoracic spine have made the morphometric analysis of the thoracic pedicle a clinical necessity for all the surgeons practicing this procedure [28,34]. Recently, the anatomical parameters of lumbar spine process have been well described [4, 27, 31], and previously studies have provided information regarding thoracic SP $[13,15,26,35,38]$. There are only few reports on the measurement of lamina space. However, a comprehensive description of the related parameters of the thoracolumbar SP and lamina space has not been reported. Meanwhile, to our knowledge, spine morphology varies across different races [19]. There have been few reports of SP morphometry in the Chinese population.

Therefore, it is essential to understand the precise anatomy of the SP and lamina space. The purpose of this study was to determine the morphometric parameters of the thoracolumbar SP and lamina space, and to provide an anatomic basis for lamina space stabilisation devices, other posterior surgery, puncture and epidural anaesthesia for the Chinese population.

\section{MATERIALS AND METHODS}

\section{Materials}

Twenty-four formalin-fixed intact adult male cadavers from the Department of Anatomy of North China University of Science and Technology ages ranged from 35 to 69 years, with a mean of 47 years. And height ranged from 160 to $175 \mathrm{~cm}$, with a mean of $168 \mathrm{~cm}$. There were no malformations and local pathological changes of the spine. Measurement instruments consist of electronic vernier calliper and compass.

\section{Measuring parameters}

The cadavers were placed in a prone position for numbering the vertebra. The posterior lumbar spine (T1-T12) (S1-S5) was exposed, and the SP, vertebral plate, and articular process were revealed (Fig. 1). Symmetric structures were measured bilaterally. Measurements were made using an electronic vernier calliper accurate to $0.01 \mathrm{~mm}$ and a compass. After each measurement, compass and electronic vernier calliper were restored the initial state of zero. All data were measured 3 times to take its average value. Statistical analysis was used to determine the average (mean), standard deviation and minimum and maximum values. The main measuring parameters were as follows:

Distance of the spine spinous process spacing, which is between the lower border of the upper adjacent SP and the upper border of the lower adjacent SP. 


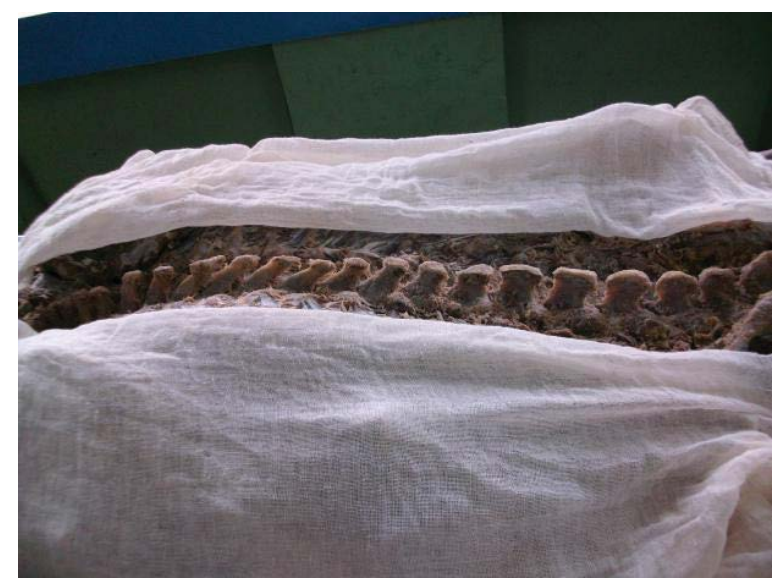

Figure 1. The thoracic and lumbar vertebral lamina and articular process after removed all muscles and ligaments.

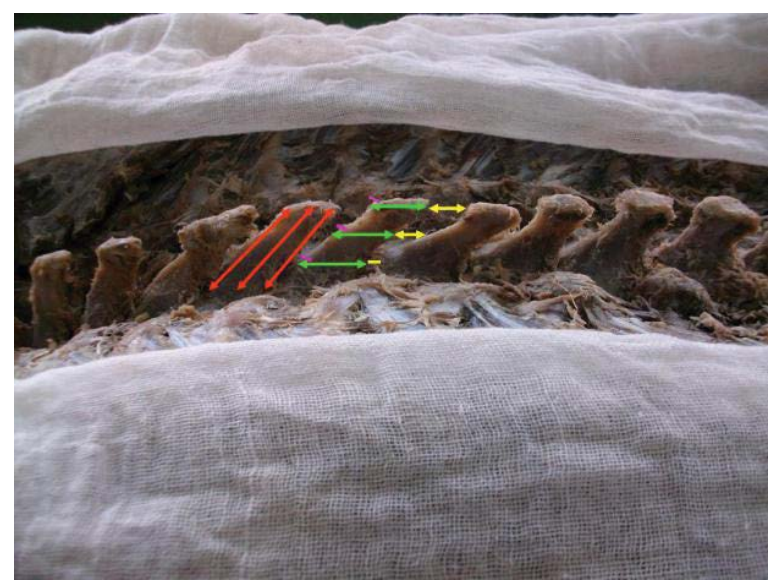

Figure 2. The distance, length, width, height of the spine spinous process in the thoracic part.

The tip distance (TD), centre distance (CD) and root distance (RD) between the adjacent SPs were measured respectively (Figs. 2, 3). The TD in this article has the same meaning as distance between the two adjacent spinous processes (DB) in other articles $[4,27]$.

Length, width, thickness of the spine spinous process: 1) The length of the SP was evaluated in the upper border $(\mathrm{UL})$, centre region $(\mathrm{CL})$ and lower border (LL) (Figs. 2, 3). 2). The width of the SP was measured in the tip border (TW), centre region (CW) and root border (RW). 3) The thickness of the SP was evaluated in the tip border (TT), centre region (CT) and root border (RT).

Inclination angle of the spine spinous process: The inclination angle of SP was measured by measuring the angles between the straight line of the upper edge (UI), the centre (Cl) and the lower edge (LI) of

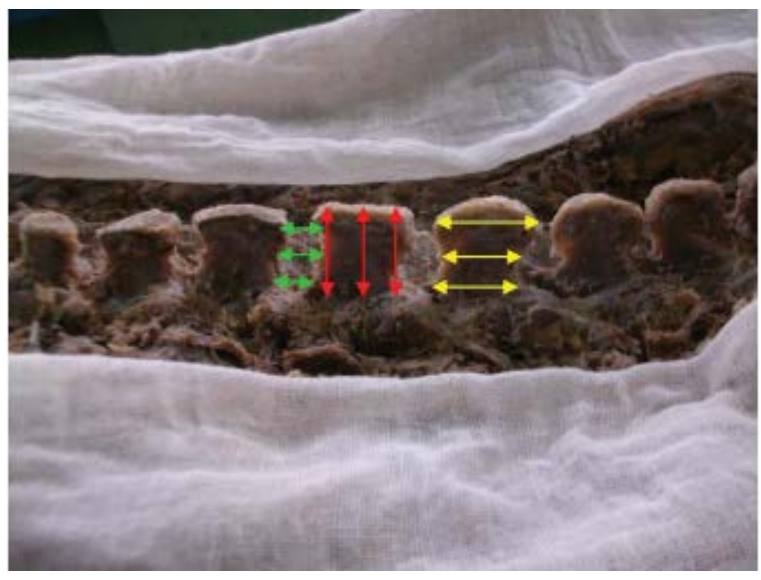

Figure 3. The distance, length, height of the spine spinous process in the lumbar part.

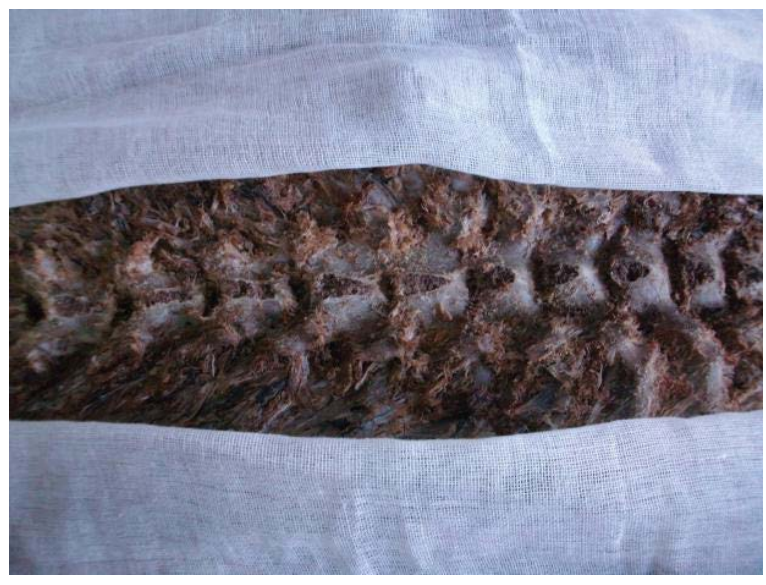

Figure 4. The lamina space after removed all the spinous process and the surrounding connective tissue.

spinous process and the tangent line of the spines, which is in the state of natural bending.

Width and height of lamina space: After removed all the SP and the surrounding connective tissue, the lamina space were fully exposed (Fig. 4). Two feet of a compass were put into the lamina space to measure the width of lamina space (WI), and the height between the upper and lower lamina from the left side $(\mathrm{LHI})$, centre region $(\mathrm{CHI})$ and right side (RHI) (Fig. 5).

Collected data were analysed and compared with other studies. The schematic diagram of relevant anatomical indicators of SP was shown at Figure 6.

\section{Ethics statement}

This study was conducted in accordance with the Declaration of Helsinki (Edinburgh 2000 revised). The Institutional Review Board of North China University of Sci- 


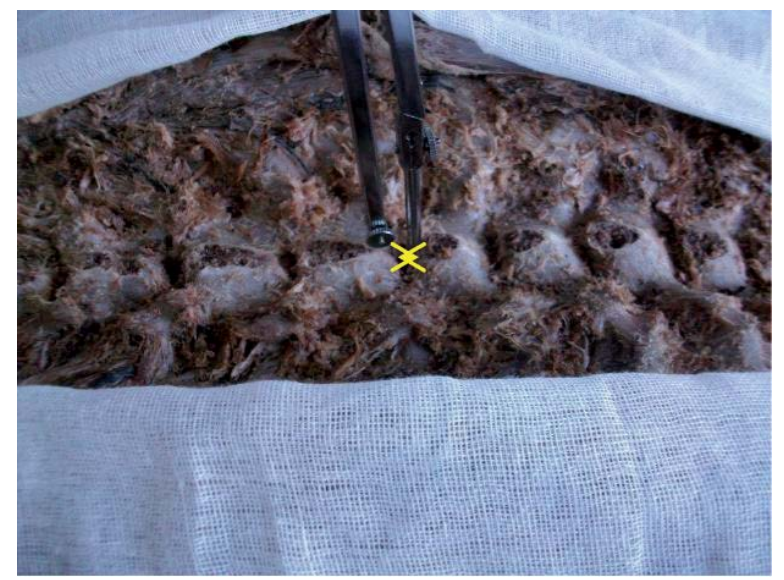

Figure 5. The height of the centre region of lamina space measured with compasses.

ence and Technology approved this study, confirm that the study was performed in accordance with relevant guidelines/regulations and informed consent was obtained from family members or their legal guardian(s).

\section{RESULTS}

\section{Distance of the spine spinous process spacing} (Table 1)

The TD > the $C D>$ the RD in each thoracic vertebra. The TD: The maximum was observed at T4 T5 $(16.80 \pm 2.34 \mathrm{~mm})$ level, and the minimum was observed at T9 T10 $(9.82 \pm 1.93 \mathrm{~mm})$ level. The CD: The maximum was observed at T11 T12 (10.98 \pm $\pm 1.91 \mathrm{~mm}$ ) level, and the minimum was observed at T5 T6 (7.56 $\pm 2.44 \mathrm{~mm})$ level. The RD: The maximum was observed at T11 T12 (9.14 $\pm 2.80 \mathrm{~mm})$ level, and the minimum was observed at T5 T6 (4.94 \pm $\pm 1.92 \mathrm{~mm}$ ) level.

The $\mathrm{CD}>$ the $\mathrm{TD}>$ the $\mathrm{RD}$ in $\mathrm{L} 1 \sim \mathrm{L} 2$ and $\mathrm{L} 2 \sim \mathrm{L} 3$ levels. The $C D>$ the $R D>$ the $T D$ in $L 3 \sim L 4, L 4 \sim L 5$ and L5 S1 levels. The TD is L2 L3 maximum $(11.94 \pm 2.01 \mathrm{~mm}), \mathrm{L} 5 \sim \mathrm{S} 1$ minimum $(6.51 \pm$ $\pm 1.47 \mathrm{~mm}$ ). The $\mathrm{CD}$ is $\mathrm{L} 1 \sim \mathrm{L} 2$ maximum (12.48 \pm $\pm 1.21 \mathrm{~mm}), \mathrm{L} 5 \sim \mathrm{S} 1$ minimum $(7.74 \pm 1.82 \mathrm{~mm})$. The $\mathrm{RD}$ is $\mathrm{L} 2 \sim \mathrm{L3}$ maximum $(10.69 \pm 2.02 \mathrm{~mm}), \mathrm{L} 4 \sim \mathrm{L} 5$ minimum $(7.46 \pm 2.26 \mathrm{~mm})$. Distance of spinous process spacing in lumbar part showed an decreasing pattern from $L 1 \sim L 2$ to $L 5 \sim S 1$.

\section{Length, width, thickness of the spine spinous process (Table 2)}

\section{The length of the spinous process}

Thoracic part: the $U L>$ the $C L>$ the $L L$ in each thoracic SP. The UL gradually increased from T1 to

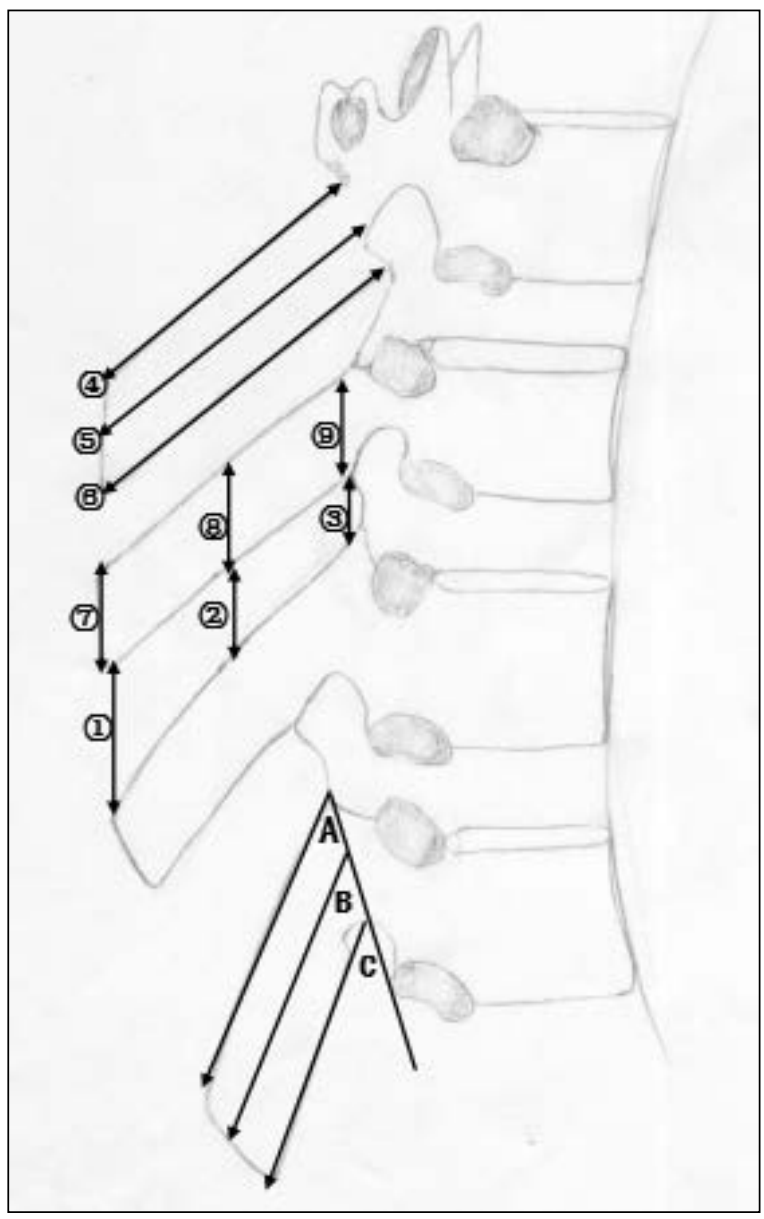

Figure 6. The schematic diagram of relevant anatomical indicators of spinous process. 1 - the tip distance (TD) of the spine spinous process spacing; 2 - the centre distance (CD) of the spine spinous process spacing; 3 - the root distance (RD) of the spine spinous process spacing; 4 - the upper length (UL) of the spine spinous process; 5 - the centre length (CL) of the spine spinous process; 6 - the lower length (LL) of the spine spinous process; 7 - the tip width (TW) of the spinous process; 8 - the centre width (CW) of the spinous process; 9 - the root width (RW) of the spinous process; $\mathrm{A}$ - the inclination angle of spinous process upper edge $(\mathrm{UI}) ; \mathrm{B}$ - the inclination angle of spinous process centre edge $(\mathrm{Cl})$; $\mathrm{C}$ - the inclination angle of spinous process lower edge (LI).

T6 to maximum value at T6 with mean of $33.38 \pm$ $\pm 2.94 \mathrm{~mm}$ and then decreased till T12 region with mean of $25.25 \pm 2.36 \mathrm{~mm}$. The $\mathrm{CL}$ is $\mathrm{T} 8(30.75 \pm 2.59$ $\mathrm{mm})$ maximum, T11 (22.52 $\pm 2.12 \mathrm{~mm})$ minimum. The LL length showed a decreasing trend from $\mathrm{T} 1$ to T12. Maximum LL was seen at T1 $(29.82 \pm 2.54 \mathrm{~mm})$ and a minimum T12 (20.35 $\pm 1.57 \mathrm{~mm})$. Lumbar part: the $U L>$ the $C L>$ the $L L$ in each lumbar SP. The length increased from $L 1$ and reached maximum value at L3 with mean of $29.17 \pm 2.35 \mathrm{~mm}$ (UL), $27.23 \pm$ $\pm 2.23 \mathrm{~mm}(\mathrm{CL}), 24.89 \pm 2.04 \mathrm{~mm}$ (LL). Then, the length decreased gradually to reach minimum value at $L 5$ with the mean of $25.12 \pm 2.37 \mathrm{~mm}$ (UL), 
Table 1. Distance of the spine spinous process spacing (mean \pm standard deviation, $\mathrm{mm}$ )

\begin{tabular}{|c|c|c|c|}
\hline Spinous process spacing & Tip distance (TD) & Centre distance (CD) & Root distance (RD) \\
\hline $\mathrm{T} 1 \sim \mathrm{T} 2$ & $10.54 \pm 1.91$ & $9.18 \pm 1.71$ & $5.82 \pm 1.29$ \\
\hline $\mathrm{T} 2 \sim \mathrm{T} 3$ & $12.66 \pm 1.97$ & $9.38 \pm 2.51$ & $5.54 \pm 1.40$ \\
\hline $\mathrm{T} 3 \sim \mathrm{T} 4$ & $14.56 \pm 1.91$ & $9.85 \pm 2.03$ & $5.67 \pm 1.62$ \\
\hline $\mathrm{T} 4 \sim \mathrm{T} 5$ & $16.80 \pm 2.34$ & $9.91 \pm 2.24$ & $5.44 \pm 1.94$ \\
\hline T5 T6 & $12.10 \pm 1.82$ & $7.56 \pm 2.44$ & $4.94 \pm 1.92$ \\
\hline $\mathrm{T} 6 \sim \mathrm{T7}$ & $12.21 \pm 2.73$ & $8.71 \pm 2.44$ & $5.99 \pm 2.53$ \\
\hline $\mathrm{T} 7 \sim \mathrm{T} 8$ & $10.82 \pm 2.04$ & $8.52 \pm 1.73$ & $6.13 \pm 2.02$ \\
\hline T8 T9 & $10.28 \pm 2.17$ & $8.03 \pm 1.69$ & $5.68 \pm 1.82$ \\
\hline T9 T10 & $9.82 \pm 1.93$ & $7.59 \pm 1.32$ & $5.65 \pm 1.61$ \\
\hline $\mathrm{T} 11 \sim \mathrm{T} 12$ & $11.99 \pm 1.90$ & $10.98 \pm 1.91$ & $9.14 \pm 2.80$ \\
\hline $\mathrm{L} 1 \sim \mathrm{L} 2$ & $11.70 \pm 1.30$ & $12.48 \pm 1.21$ & $10.23 \pm 1.44$ \\
\hline $\mathrm{L} 2 \sim \mathrm{L} 3$ & $11.94 \pm 2.01$ & $12.38 \pm 1.61$ & $10.69 \pm 2.02$ \\
\hline $\mathrm{L} 3 \sim \mathrm{L} 4$ & $9.29 \pm 1.47$ & $10.27 \pm 2.32$ & $9.72 \pm 2.42$ \\
\hline$L 4 \sim \mathrm{L} 5$ & $6.76 \pm 1.63$ & $7.77 \pm 1.12$ & $7.46 \pm 2.26$ \\
\hline $\mathrm{L} 5 \sim \mathrm{S} 1$ & $6.51 \pm 1.47$ & $7.74 \pm 1.82$ & $7.51 \pm 2.28$ \\
\hline
\end{tabular}

Table 2. Length, width and thickness of spine spinous process (mean \pm standard deviation, $\mathrm{mm}$ )

\begin{tabular}{|c|c|c|c|c|c|c|c|c|c|}
\hline \multirow[t]{2}{*}{ T1 L5 } & \multicolumn{3}{|c|}{ Length } & \multicolumn{3}{|c|}{ Width } & \multicolumn{3}{|c|}{ Thickness } \\
\hline & Upper (UL) & Centre (CL) & Lowe & Tip (TW) & Centre (CW) & Root (RW) & Tip (TT) & Centre (CT) & Root (RT) \\
\hline $\mathrm{T} 1$ & $32.48 \pm 3.28$ & $29.69 \pm 2.61$ & $29.82 \pm 2.54$ & $11.38 \pm 0.99$ & $10.99 \pm 0.80$ & $15.82 \pm 1.42$ & $7.57 \pm 1.03$ & $7.68 \pm 1.00$ & $9.76 \pm 1.33$ \\
\hline $\mathrm{T} 2$ & $31.53 \pm 2.96$ & $29.86 \pm 2.60$ & $28.67 \pm 2.50$ & $11.72 \pm 1.10$ & $12.95 \pm 1.38$ & $16.12 \pm 1.53$ & $.52 \pm 0.94$ & $7.38 \pm 0.97$ & $12.14 \pm 1.00$ \\
\hline T3 & $3.03 \pm 3.74$ & $0.35 \pm 2.68$ & $28.47 \pm 2.38$ & $3.30 \pm 0.84$ & $14.11 \pm 1.42$ & $17.92 \pm 1.55$ & $.47 \pm 0.77$ & $6.88 \pm 0.93$ & $=1.22$ \\
\hline T4 & $31.43 \pm 3.21$ & $29.51 \pm 2.75$ & $27.55 \pm 2.40$ & $13.83 \pm 0.91$ & $15.05 \pm 0.86$ & $18.52 \pm 1.28$ & $5.18 \pm 0.73$ & $6.41 \pm 0.92$ & $12.6 \mathrm{~s}$ \\
\hline T5 & $32.98 \pm 2.44$ & $27.50 \pm 2.46$ & $28.39 \pm 2.53$ & $28.39 \pm 2.53$ & $15.73 \pm 0.80$ & $19.80 \pm 1.64$ & $5.07 \pm 0.62$ & $6.79 \pm 1.01$ & $12.05 \pm 1.42$ \\
\hline T6 & $33.38 \pm 2.94$ & $29.94 \pm 2.86$ & $28.24 \pm 2.40$ & $28.24 \pm 2.40$ & $16.07 \pm 1.09$ & $20.14 \pm 1.60$ & $4.76 \pm 0.80$ & $6.36 \pm 1.01$ & $13.67 \pm 1.52$ \\
\hline $\mathrm{T7}$ & $31.55 \pm 2.77$ & $29.64 \pm 2.42$ & $27.80 \pm 3.08$ & $27.80 \pm 3.08$ & $15.39 \pm 1.79$ & $20.36 \pm 1.54$ & $4.41 \pm 0.77$ & $5.73 \pm 0.99$ & $10.13 \pm 2.05$ \\
\hline T8 & $32.65 \pm 2.74$ & $30.75 \pm 2.59$ & $25.15 \pm 2.60$ & $25.15 \pm 2.60$ & $15.62 \pm 1.21$ & $20.42 \pm 1.56$ & $4.89 \pm 0.67$ & $5.95 \pm 0.84$ & $8.43 \pm 0.78$ \\
\hline T9 & $31.50 \pm 2.92$ & $23.07 \pm 2.33$ & $22.90 \pm 2.69$ & $22.90 \pm 2.69$ & $16.93 \pm 0.91$ & $22.18 \pm 1.52$ & $5.26 \pm 0.74$ & $6.22 \pm 0.73$ & $7.87 \pm 1.69$ \\
\hline $\mathrm{T} 10$ & $29.62 \pm 2.80$ & $23.09 \pm 2.52$ & $21.53 \pm 2.87$ & $21.53 \pm 2.87$ & $17.37 \pm 0.84$ & $22.56 \pm 1.50$ & $6.03 \pm 1.10$ & $6.93 \pm 0.85$ & \pm 1.70 \\
\hline T11 & $25.49 \pm 2.90$ & $22.51 \pm 2.12$ & $20.77 \pm 2.15$ & $20.77 \pm 2.15$ & $18.56 \pm 0.90$ & $22.60 \pm 1.30$ & $6.50 \pm 0.80$ & $7.87 \pm 0.86$ & $10.98 \pm 1.28$ \\
\hline T12 & $25.25 \pm 2.36$ & $23.13 \pm 2.44$ & $20.35 \pm 1.57$ & $20.35 \pm 1.57$ & $19.88 \pm 1.53$ & $23.80 \pm 0.81$ & $7.03 \pm 1.11$ & $8.50 \pm 0.98$ & $12.14 \pm 1.45$ \\
\hline L1 & $25.59 \pm 2.13$ & $25.52 \pm 2.85$ & $21.66 \pm 2.34$ & $22.68 \pm 2.17$ & $21.99 \pm 1.10$ & $24.94 \pm 1.48$ & $7.70 \pm 1.26$ & 7.75 & $9.74 \pm 1.51$ \\
\hline L2 & $27.09 \pm 2.10$ & $25.78 \pm 2.52$ & $24.05 \pm 2.22$ & $22.23 \pm 0.72$ & $22.48 \pm 1.49$ & $25.19 \pm 1.30$ & $7.16 \pm 1.32$ & $7.28 \pm 0.95$ & $11.43 \pm 1.76$ \\
\hline L3 & $29.17 \pm 2.35$ & $27.23 \pm 2.23$ & $24.89 \pm 2.04$ & $23.68 \pm 1.41$ & $23.56 \pm 1.36$ & $25.83 \pm 0.96$ & $7.12 \pm 1.14$ & $7.47 \pm 0.51$ & $9.93 \pm 1.51$ \\
\hline L4 & $27.88 \pm 2.44$ & $26.33 \pm 2.32$ & $22.68 \pm 2.84$ & $23.59 \pm 1.44$ & $21.26 \pm 0.91$ & $23.35 \pm 1.71$ & $7.63 \pm 1.19$ & $7.39 \pm 0.67$ & $11.43 \pm 0.89$ \\
\hline L5 & $25.12 \pm 2.37$ & $23.76 \pm 2.82$ & $20.80 \pm 2.75$ & $19.54 \pm 0.78$ & $17.91 \pm 1.85$ & $17.20 \pm 1.72$ & $7.25 \pm 0.92$ & $8.11 \pm 0.83$ & $10.69 \pm 1.80$ \\
\hline
\end{tabular}

$23.76 \pm 2.82 \mathrm{~mm}(\mathrm{CL}), 20.80 \pm 2.75 \mathrm{~mm}(\mathrm{LL})$. Upper border length of the lower spine $>$ lower border length of the upper adjacent spine.

\section{The width of the spinous process}

Thoracic part: RW > CW > TW in each thoracic vertebra approximately, and the width mentioned showed an increasing trend from $\mathrm{T} 1$ with the mean of $11.38 \pm 0.99 \mathrm{~mm}$ (TW), $10.99 \pm 0.80 \mathrm{~mm}(\mathrm{CW})$, $15.81 \pm 1.42 \mathrm{~mm}(\mathrm{RW})$ to T12 with the mean of $20.28 \pm 1.57 \mathrm{~mm}$ (TW), $19.88 \pm 1.53 \mathrm{~mm}(\mathrm{CW})$, $23.80 \pm 0.81 \mathrm{~mm}(\mathrm{RW})$. Lumbar part: Maximum width was seen at $L 3$ with the mean of $23.68 \pm 1.41 \mathrm{~mm}$ (TW), $23.56 \pm 1.36 \mathrm{~mm}(\mathrm{CW}), 25.83 \pm 0.96 \mathrm{~mm}(\mathrm{RW})$ and 
Table 3. The inclination angle of spinous process (mean \pm standard deviation, ${ }^{\circ}$ )

\begin{tabular}{lccc}
\hline T1 T12 & Upper edge inclination angle (UI) & Centre edge inclination angle (Cl) & Lower edge inclination angle (LI) \\
\hline T1 & $61.20 \pm 2.17$ & $62.63 \pm 3.38$ & $67.70 \pm 3.50$ \\
T2 & $58.33 \pm 3.50$ & $60.50 \pm 3.12$ & $64.80 \pm 3.77$ \\
T3 & $54.30 \pm 4.14$ & $57.38 \pm 3.46$ & $59.20 \pm 3.03$ \\
T4 & $49.40 \pm 1.95$ & $52.50 \pm 3.78$ & $55.00 \pm 3.16$ \\
T5 & $40.57 \pm 3.74$ & $45.83 \pm 3.66$ & $48.40 \pm 2.70$ \\
T6 & $38.29 \pm 3.25$ & $41.50 \pm 3.82$ & $43.67 \pm 3.43$ \\
T7 & $37.25 \pm 3.15$ & $38.14 \pm 2.48$ & $39.50 \pm 3.70$ \\
T8 & $40.89 \pm 2.57$ & $41.44 \pm 3.28$ & $42.14 \pm 3.34$ \\
T9 & $42.63 \pm 3.89$ & $45.38 \pm 3.93$ & $52.88 \pm 1.96$ \\
T10 & $57.44 \pm 2.46$ & $61.11 \pm 2.67$ \\
T11 & $51.67 \pm 3.93$ & $71.38 \pm 3.50$ & $78.00 \pm 2.65$ \\
T12 & $59.50 \pm 2.33$ & $78.20 \pm 3.70$ & $86.50 \pm 2.38$ \\
\hline
\end{tabular}

a minimum L5 with the mean of $19.54 \pm 0.78 \mathrm{~mm}$ (TW), $17.91 \pm 1.85 \mathrm{~mm}$ (CW), $17.20 \pm 1.72 \mathrm{~mm}(\mathrm{RW})$.

\section{The thickness of the spinous process}

Tip thickness (TT) > centre thickness $(\mathrm{CT})>$ root thickness (RT) in each thoracic and lumbar vertebra. Thoracic part: the maximum TT is T1 $(7.57 \pm 1.03 \mathrm{~mm})$, $\mathrm{T} 7$ minimum $(4.41 \pm 0.77 \mathrm{~mm})$, The maximum $\mathrm{CT}$ is $\mathrm{T} 12(8.50 \pm 0.98 \mathrm{~mm}), \mathrm{T} 7$ minimum $(5.73 \pm 0.99 \mathrm{~mm})$. The maximum RT is T6 (13.67 $\pm 1.52 \mathrm{~mm})$, T9 minimum $(7.87 \pm 1.69 \mathrm{~mm})$. Lumbar part: Maximum TT was seen at $\mathrm{L} 1$ with the mean of $7.70 \pm 1.26 \mathrm{~mm}$, and a minimum $L 3$ with the mean of $7.12 \pm 1.14 \mathrm{~mm}$. Maximum CT was seen at L5 with the mean of $8.11 \pm$ $\pm 0.83 \mathrm{~mm}$, and a minimum L2 with the mean of $7.28 \pm 0.95 \mathrm{~mm}$. Maximum RT was seen at $\mathrm{L} 2$ with the mean of $11.43 \pm 1.76 \mathrm{~mm}$, and a minimum L1 with the mean of $9.74 \pm 1.51 \mathrm{~mm}$.

\section{Inclination angle of the spine spinous process (Table 3)}

The inclination angle gradually decreased from T1 to T7 to minimum value at T7 with mean of $37.25 \pm$ $\pm 3.15^{\circ}(\mathrm{UI}), 38.14 \pm 2.48^{\circ}(\mathrm{Cl})$ and $86.50 \pm 2.38^{\circ}$ (LI) and then increased till T12 region with mean of $74.29 \pm 3.50^{\circ}(\mathrm{UI}), 78.20 \pm 3.70^{\circ}(\mathrm{Cl})$ and $39.50 \pm$ $\pm 3.70^{\circ}(\mathrm{LI})$. And the lower edge inclination angle (LI) $>$ the centre edge inclination angle $(\mathrm{Cl})>$ the upper edge inclination angle (UI) in each thoracic vertebra. Generally, the inclination angle of lumbar spine is basic 90 degrees, which is of little significance. Thus we did not measure the inclination angle of lumbar spine.

\section{Width and height of lamina space (Table 4)}

The width of lamina space: For thoracic part, the data became shorter gradually from T1 T2 (11.73 \pm $\pm 2.51 \mathrm{~mm})$ to $\mathrm{T} 5 \sim \mathrm{T} 6(9.63 \pm 3.63 \mathrm{~mm})$, and then increased till to $\mathrm{T} 11 \sim \mathrm{T} 12(10.70 \pm 4.67 \mathrm{~mm})$. For lumbar part, the width of lamina space increased from T12 L1 $(12.18 \pm 1.43 \mathrm{~mm})$ to $\mathrm{L} 5 \sim \mathrm{S} 1$ (15.64 \pm $\pm 1.73 \mathrm{~mm}$ ). Among them, the increment of L5 S1 width was the largest, and larger about $2 \mathrm{~mm}$ than L4 L5 width.

The height of lamina space: In the thoracic vertebrae, the maximum height of centre region was observed at T11 T12 (7.47 $\pm 2.78 \mathrm{~mm})$ and the minimum mean value was observed at T3 T4 (5.20 \pm $\pm 1.77 \mathrm{~mm}$ ). Since the thoracic space height is small, the left and right sides are difficult to measure, so this study did not measure this two indicators. In the lumbar vertebrae, the height of the lamina space was gradually increased from $T 12 \sim L 1$ to $L 5 \sim S 1$. The height of the centre region was greater than the height of the left and right sides, and there was no significant difference between the heights of the left and right sides. The centre height increased from $9.68 \pm 1.76 \mathrm{~mm}$ to $11.88 \pm 1.78 \mathrm{~mm}$, the left height increased from $6.91 \pm 1.16 \mathrm{~mm}$ to $7.79 \pm 1.19 \mathrm{~mm}$, and the right height increased from $7.04 \pm 0.92 \mathrm{~mm}$ to $7.79 \pm 1.06 \mathrm{~mm}$.

\section{DISCUSSION}

At present, lumbar spine process-related indicators have been reported [4, 27, 31], and there are a few reports on the measurement of thoracic SPs $[13,15,26,35,38]$. There are also some studies 
Table 4. The width and height of lamina space (mean \pm standard deviation, $\mathrm{mm}$ )

\begin{tabular}{lcccc}
\hline Lamina space & Width (WI) & & Height & Right side (RHI) \\
\cline { 2 - 4 } & & Centre region (CHI) & Left side (LH) & - \\
\hline T1 T2 & $11.73 \pm 2.51$ & $6.20 \pm 2.49$ & - & - \\
T2 T3 & $11.53 \pm 2.87$ & $6.01 \pm 2.25$ & - & - \\
T3 T4 & $10.20 \pm 3.44$ & $5.20 \pm 1.77$ & - & - \\
T4 T5 & $10.62 \pm 3.84$ & $5.49 \pm 2.10$ & - & - \\
T5 T6 & $9.63 \pm 3.63$ & $5.65 \pm 2.03$ & - & - \\
T6 T7 & $10.92 \pm 4.95$ & $5.32 \pm 2.14$ & - & - \\
T7 T8 & $10.80 \pm 4.94$ & $5.91 \pm 2.67$ & - & - \\
T8 T9 & $10.33 \pm 4.28$ & $5.52 \pm 2.17$ & - & - \\
T9 T10 & $10.20 \pm 4.54$ & $6.00 \pm 2.57$ & - & $7.04 \pm 0.92$ \\
T10 T11 & $9.72 \pm 5.12$ & $6.92 \pm 4.28$ & $7.18 \pm 0.66$ \\
T11 T12 & $10.70 \pm 4.67$ & $7.47 \pm 2.78$ & $7.29 \pm 0.56$ \\
T12 L1 & $12.18 \pm 1.43$ & $9.68 \pm 1.76$ & $7.91 \pm 1.16$ & $7.44 \pm 0.84$ \\
L1 L L2 & $12.81 \pm 2.12$ & $10.24 \pm 0.91$ & $7.06 \pm 0.66$ & $7.56 \pm 0.88$ \\
L2 L3 & $12.92 \pm 1.80$ & $11.19 \pm 0.83$ & $7.30 \pm 0.77$ & $7.79 \pm 1.06$ \\
L3 L4 & $13.10 \pm 1.91$ & $11.20 \pm 1.37$ & $7.43 \pm 0.57$ & $7.60 \pm 0.87$ \\
L4 L5 & $13.71 \pm 1.37$ & $11.32 \pm 0.77$ & $7.79 \pm 1.19$ & - \\
L5 S1 & $15.64 \pm 1.73$ & $11.88 \pm 1.78$ & & - \\
\hline
\end{tabular}

that report measurements of thoracic and lumbar spine-related indicators separately, which are not comprehensive enough. In the morphology of lumbar spine processes studied by Cai et al. [4], only the distance, height, thickness of the upper, middle and lower borders of the lumbar spine were studied. Bo Ran et al. [27] performed lumbar spine morphology in Chinese population by three-dimensional computed tomography reconstruction. Kiranpreet Kaur et al. [15] only reported the inclination angle of the thoracic spine. Jeremy D. Shaw et al. [31] analysed the length, width, height, slope and tail morphology of lumbar spine, but did not measure the distance between SPs spacing. In our study, more relevant indicators of thoracolumbar spine and lamina space were measured based on intact corpses, which include distance of SPs spacing, SP length, width, thickness, and inclination angle of the SP, and width, height (centre, left and right) of the lamina space.

The experimental results of the SP spacing distance in each thoracic vertebra show that the tip distance $>$ the centre distance $>$ the root distance. The maximum of the tip distance was observed at $\mathrm{T} 4 \sim \mathrm{T} 5$, and the minimum was T9 T10. The maximum of the centre distance was observed at $\mathrm{T} 11 \sim \mathrm{T} 12$, and the minimum was T5 T6. The maximum of the root distance was observed at $\mathrm{T} 11 \sim \mathrm{T} 12$, and the minimum was T5 T6. Thus, the thoracic SP spacing distance is wedge-shaped. In the sagittal plane, the front is high and the back is low. To avoid damage caused by stress concentration of the implant, the internal fixation device should have a wedge-shaped structure, which is high in front and low in back. The maximum tip distance of lumbar SP spacing was noted at L2 L3, while the minimum tip distance was L5 S1. The maximum centre distance of lumbar SP spacing was noted at $L 1 \sim L 2$, then it gradually decreased to the minimum root distance $(\mathrm{L} 5 \sim \mathrm{S} 1)$. The maximum root distance of lumbar SP spacing was noted at $L 2 \sim L 3$, while the minimum root distance was $L 4 \sim \mathrm{L} 5$. The above results are different from the data of Cai et al. [4], which considered that distance between lumbar spines was gradually decreased from $L 1 \sim L 2$ to $L 5 \sim$ S1. And our results differ from Bo Ran [27] and others' opinion, which hold that the distance between lumbar spines was gradually decreased from $L 1 \sim L 2$ to $L 4 \sim L 5$, and then increased from $L 4 \sim L 5$ to $L 5 \sim S 1$. Cai et al. [4] and Bo Ran et al. [27] measured the above parameter at only one point, while our study performed it at three points (tip, centre and root), which is more comprehensive and detailed. Recently, numerous lamina space implants have been introduced and have shown favourable outcomes in the treatment of degenerative disc disease, herniated nucleus pul- 
posus, lumbar spinal stenosis, lumbar instability, and degenerative lumbar spondylolisthesis $[2,7,23,32$, 37]. Nevertheless, there are still complications with lamina space implants. Therefore, choosing optimal sizes of implants is important to avoid unnecessary complications and the size of the device should be carefully evaluated $[3,14]$. The data in this study can help clinicians grasp the optimal size of the implant, which is one way to reduce complications.

By measuring the length of the spine SP, we can learn that the upper border $>$ the centre region $>$ the lower border in each thoracic and lumbar SP. In the lumbar part, L3 is the maximum and L5 is the minimum, which is consistent with the conclusions drawn by Cai et al. [4] and Bo Ran et al. [27]. Usually, spinal lesions are treated with a posterior approach, where surgeons can remove the posterior part of the spine in order to expand the field of vision. In this process, the knowledge of the length, width and thickness of the spine SPs provides a comprehensive reference for the type of instrument chosen and the location of the occlusal site. With known length, width, and thickness, we can estimate the size of the SPs of the spine, which is important for lumbar interbody fusion [12]. In addition, the designing of SP internal fixation provides an equivalent pain/ function improvement compared to conventional posterior-lumbar-interbody-fusion pedicle screw fixation, reducing the number of hospitalisations and operations [5]. So, the above indicators are also important reference indicators for the design of SP internal fixation devices.

The SP of the thoracic spine is obliquely posteriorly downward, but the specific values of the inclination angle are rare. Kiranpreet Kaur et al. [15] used the scanner software to measure the SP angle of volunteers, showing that the SP angle increased from $\mathrm{T} 1$ and reached maximum value at $\mathrm{T} 6$ level, then decreased gradually and reached minimum value at $\mathrm{T} 12$ level. In our study, the intact cadaver specimens were used to expose the SPs of the thoracic spine. The inclination angle of SP was measured by measuring the angles between the straight line of the upper edge, the centre and the lower edge of SP and the tangent line of the spines, which is in the state of natural bending. The inclination angle gradually decreased from $\mathrm{T} 1$ to $\mathrm{T} 7$ to minimum value and then increased till $\mathrm{T} 12$ region, which is slightly different from result of the above document. In clinical application, the setting of the inclination angle of the upper and lower edges of the SP internal fixation needs to refer to the inclination angle of the SP to be inserted into the internal fixation.

Laminar space plays an important role in spinal surgery. Clinically, most of the procedures such as disc herniation, spinal stenosis, and intraspinal schwannomas need to be taken from the lamina space. Therefore, the study of anatomical parameters of the laminar space can provide a basis for relevant clinical operations. For example, in the treatment of disc herniation, percutaneous endoscopic laser-assisted discectomy (PELD) has been widely used in clinical practice in recent years $[1,20,22]$. The outer diameter of the required endoscope is $7.5 \mathrm{~mm}$, and the working channel is at the centre of the lamina space. According to the data obtained in this experiment, it is not difficult to implement PELD in L4 L5 and L5 S1. For another example, spinal stenosis is also a common disease in the spinal region, which is due to a cascade of degenerative processes starting with degeneration of posterior annulus to disc herniation and dehydration, then to loss of disc height, overriding of the facets, and/or infolding of ligamentum flavum, and ultimately stenosis. This condition occurs as a result of age-related spinal degeneration, particularly in the lamina space disc and ligamentum flavum. Common symptoms include radicular pain and neurogenic claudication, which is mainly treated by surgical intervention $[6,24,36]$. This requires some anatomical parameters of the lamina space to guide the operation. In addition, the removal of schwannomas in the spinal canal also requires from lamina space [21, 25], whose anatomical parameters are particularly important. In addition to the surgical approach, the measurement of the width and height of the lamina space provides an anatomical basis for the intraspinal puncture approach. According to reports in the literature, emergency doctors can quickly obtain lumbar anatomical markers by ultrasound [10], These data can also provide reference and experience for clinicians to help clinical first aid and other treatments.

\section{CONCLUSIONS}

More related indicators of the thoracolumbar spine spinous process and intervertebral space were measured based on intact cadavers in our study, including the distance, length, width, thickness, inclination angle of the spinous process and the width, height of the intervertebral space, to provide com- 
prehensive anatomic basis for thoracolumbar spine design of internal fixation, posterior surgery, puncture and epidural anaesthesia for the Chinese population.

\section{Acknowledgements}

We thank Dr. Guangyao Song for his careful reading of the manuscript and helpful suggestions.

\section{Conflict of interest: None declared}

\section{REFERENCES}

1. Ahn Y, Lee SH, Park WM, et al. Percutaneous endoscopic lumbar discectomy for recurrent disc herniation: surgical technique, outcome, and prognostic factors of 43 consecutive cases. Spine (Phila Pa 1976). 2004; 29(16): E326-E332, doi: 10.1097/01.brs.0000134591.32462.98, indexed in Pubmed: 15303041.

2. Bono CM, Vaccaro AR. Lamina space process devices in the lumbar spine. J Spinal Disord Tech. 2007; 20: 255-261.

3. Bowers C, Amini A, Dailey AT, et al. Dynamic interspinous process stabilization: review of complications associated with the X-Stop device. Neurosurg Focus. 2010; 28(6): E8, doi: 10.3171/2010.3.FOCUS1047, indexed in Pubmed: 20568923.

4. Cai B, Ran B, Li Q, et al. A morphometric study of the lumbar spinous process in the Chinese population. Braz J Med Biol Res. 2015; 48(1): 91-95, doi: 10.1590/1414431x20144164, indexed in Pubmed: 25493388.

5. Castilleja A, Broomall PA. Pedicle screws vs minimally invasive posterior-lumbar-interbody-fusion with spinous process fixation in the workers compensation population: early outcomes with minimum one year follow up. Orthopaedic Surg. 2014: E123-E124.

6. Chen PGC, Daubs MD, Berven S, et al. Surgery for degenerative lumbar scoliosis: the development of appropriateness criteria. Spine (Phila Pa 1976). 2016; 41(10): 910-918, doi: 10.1097/BRS.0000000000001392, indexed in Pubmed: 26679874

7. Chiu JC. lamina space process decompression (IPD) system (X-STOP) for the treatment of lumbar spinal stenosis. Surg Technol Int. 2006; 15: 265-275.

8. Dommisse GF. The blood supply of the spinal cord. J Bone Joint Surg. 1974; 56-B(2): 225-235, doi: 10.1302/0301-620x.56b2.225.

9. Drake RL. ed. In: Textbook of Gray's Anatomy for Students 1st ed. Churchill Livingstone Publications, Philadelphia 2005: 33-63.

10. Ferre RM, Sweeney TW. Emergency physicians can easily obtain ultrasound images of anatomical landmarks relevant to lumbar puncture. Am J Emerg Med. 2007; 25(3): 291-296, doi: 10.1016/j.ajem.2006.08.013, indexed in Pubmed: 17349903.

11. Gazzeri R, Galarza M, Alfieri A. Controversies about interspinous process devices in the treatment of degenerative lumbar spine diseases: past, present, and future. Biomed Res Int. 2014; 2014: 975052, doi: 10.1155/2014/975052, indexed in Pubmed: 24822224.

12. Hu MW, Liu ZL, Zhou Y, et al. Posterior lumbar interbody fusion using spinous process and laminae. J Bone Joint Surg Br.
2012; 94(3): 373-377, doi: 10.1302/0301-620X.94B3.27629, indexed in Pubmed: 22371546.

13. Husted DS, Haims AH, Fairchild TA, et al. Morphometric comparison of the pedicle rib unit to pedicles in the thoracic spine. Spine (Phila Pa 1976). 2004; 29(2): 139-146, doi: 10.1097/01.BRS.0000105537.49674.A3, indexed in Pubmed: 14722404.

14. Ihm EH, Han InBo, Shin DAh, et al. Spinous process morphometry for interspinous device implantation in Korean patients. World Neurosurg. 2013; 79(1): 172-176, doi: 10.1016/j.wneu.2011.04.027, indexed in Pubmed: 22120390.

15. Kaur K, Singh R, Prasath V, et al. Computed tomographic-based morphometric study of thoracic spine and its relevance to anaesthetic and spinal surgical procedures. J Clin Orthop Trauma. 2016; 7(2): 101-108, doi: 10.1016/j. jcot.2015.12.002, indexed in Pubmed: 27182147.

16. Kim D, Albert T. Interspinous process spacers. J Am Acad Orthop Surg. 2007; 15(4): 200-207, doi: 10.5435/00124635200704000-00003.

17. Kim HoJ, Bak KH, Chun HJ, et al. Posterior interspinous fusion device for one-level fusion in degenerative lumbar spine disease : comparison with pedicle screw fixation: preliminary report of at least one year follow up. J Korean Neurosurg Soc. 2012; 52(4): 359-364, doi: 10.3340/ jkns.2012.52.4.359, indexed in Pubmed: 23133725.

18. Leahy JC, Mathias KJ, Hukins DW, et al. Design of spinous process hooks for flexible fixation of the lumbar spine. Proc Inst Mech Eng H. 2000; 214(5): 479-487, doi: 10.1243/0954411001535507, indexed in Pubmed: 11109855.

19. Lee HM, Kim NH, Kim HJ, et al. Morphometric study of the lumbar spinal canal in the Korean population. Spine (Phila Pa 1976). 1995; 20(15): 1679-1684, doi: 10.1097/00007632-199508000-00006, indexed in Pubmed: 7482017.

20. Lee SH, Kang HS. Percutaneous endoscopic laser annuloplasty for discogenic low back pain. World Neurosurg. 2010; 73(3): 198-206; discussion e33, doi: 10.1016/j. surneu.2009.01.023, indexed in Pubmed: 20860958.

21. Lee SE, Jahng TA, Kim HJ. Different surgical approaches for spinal schwannoma: a single surgeon's experience with 49 consecutive cases. World Neurosurg. 2015; 84(6): 1894-1902, doi: 10.1016/j.wneu.2015.08.027, indexed in Pubmed: 26325210.

22. Lühmann D, Burkhardt-Hammer T, Borowski C, et al. Minimally invasive surgical procedures for the treatment of lumbar disc herniation. GMS Health Technol Assess. 2005; 1: Doc07, indexed in Pubmed: 21289928.

23. Mariottini A, Pieri $S$, Giachi $S$, et al. Preliminary results of a soft novel lumbar intervertebral prothesis (DIAM) in the degenerative spinal pathology. Acta Neurochir Suppl. 2005; 92: 129-131, doi: 10.1007/3-211-27458-8_28, indexed in Pubmed: 15830984.

24. Miscusi M, Trungu S, Forcato S, et al. Long-term clinical outcomes and quality of life in elderly patients treated with interspinous devices for lumbar spinal stenosis. J Neurol Surg A Cent Eur Neurosurg. 2018; 79(2): 139-144, doi: 10.1055/s-0037-1604280, indexed in Pubmed: 28750451.

25. Onen MR, Simsek M, Naderi S. Alternatives to surgical approach for giant spinal schwannomas. Neuro- 
sciences (Riyadh). 2016; 21(1): 30-36, doi: 10.17712/ nsj.2016.1.20150242, indexed in Pubmed: 26818164.

26. Panjabi MM, O'Holleran JD, Crisco JJ, et al. Complexity of the thoracic spine pedicle anatomy. Eur Spine J. 1997; 6(1): 19-24, doi: 10.1007/BF01676570, indexed in Pubmed: 9093823 .

27. Ran B, Li Q, Yu B, et al. Morphometry of lumbar spinous process via three dimensional CT reconstruction in a Chinese population. Int J Clin Exp Med. 2015; 8(1): 1129-1136, indexed in Pubmed: 25785103.

28. Richardson J, Groen G. Applied epidural anatomy. Continuing Education Anaesthesia Critical Care Pain. 2005; 5(3): 98-100, doi: 10.1093/bjaceaccp/mki026.

29. Romanes GJ. ed. 15th ed. Cunninghams Manual of Practical Anatomy. vol. II. Oxford University Press, New York 1996: 3-82.

30. Sénégas J. Mechanical supplementation by non-rigid fixation in degenerative intervertebral lumbar segments: the Wallis system. Eur Spine J. 2002; 11 Suppl 2: S164-S169, doi: 10.1007/s00586-002-0423-9, indexed in Pubmed: 12384740.

31. Shaw JD, Shaw DL, Cooperman DR, et al. Characterization of lumbar spinous process morphology: a cadaveric study of 2,955 human lumbar vertebrae. Spine J. 2015; 15(7): 1645-1652, doi: 10.1016/j.spinee.2015.03.007, indexed in Pubmed: 25777742.

32. Shim C, Park S, Lee SH, et al. Biomechanical evaluation of an interspinous stabilizing device, locker. Spine. 2008; 33(22): E820-E827, doi: 10.1097/brs.0b013e3181894fb1.

33. Singh R, Srivastva SK, Prasath CS, et al. Morphometric measurements of cadaveric thoracic spine in Indian population and its clinical applications. Asian Spine J. 2011;
5(1): 20-34, doi: 10.4184/asj.2011.5.1.20, indexed in Pubmed: 21386943.

34. Suk SI, Kim WJ, Lee SM, et al. Thoracic pedicle screw fixation in spinal deformities: are they really safe? Spine (Phila Pa 1976). 2001; 26(18): 2049-2057, doi: 10.1097/00007632-200109150-00022, indexed in Pubmed: 11547207.

35. Ugur HC, Attar A, Uz A, et al. Thoracic pedicle: surgical anatomic evaluation and relations. J Spinal Disord. 2001; 14(1): 39-45, doi: 10.1097/00002517-200102000-00007, indexed in Pubmed: 11242273.

36. Weinstein JN, Tosteson TD, Lurie JD, et al. Surgical versus nonoperative treatment for lumbar spinal stenosis fouryear results of the Spine Patient Outcomes Research Trial. Spine (Phila Pa 1976). 2010; 35(14): 1329-1338, doi: 10.1097/BRS.0b013e3181e0f04d, indexed in Pubmed: 20453723

37. Yano S, Hida K, Seki T, et al. A new ceramic interspinous process spacer for lumbar spinal canal stenosis. Operative Neurosurgery. 2008; 63(suppl_1): ONS108-ONS114, doi: 10.1227/01.neu.0000310693.86660.d3.

38. Zindrick MR, Knight GW, Sartori MJ, et al. Pedicle morphology of the immature thoracolumbar spine. Spine (Phila Pa 1976). 2000; 25(21): 2726-2735, doi: 10.1097/00007632200011010-00003, indexed in Pubmed: 11064516.

39. Zucherman JF, Hsu KY, Hartjen CA, et al. A prospective randomized multi-center study for the treatment of lumbar spinal stenosis with the X STOP interspinous implant: 1-year results. Eur Spine J. 2004; 13(1): 22-31, doi: 10.1007/s00586-003-0581-4, indexed in Pubmed: 14685830 